\title{
Molecular Dynamics Simulations on Melting Properties of Free Icosahedral Copper Clusters
}

\author{
Jeong Won Kang and Ho Jung Hwang \\ Institute of Science and Technology, Department of Electronic Engineering, Chung-Ang \\ University, Seoul 156-756, Korea \\ E-mail :kok@semilab3.ee.cau.ac.kr
}

(Received 28 October 2002, Accepted 6 January 2003)

\begin{abstract}
We have studied the size confinement effect on the properties of melting-like transition of small icosahedral copper clusters using a classical molecular dynamics simulation based on a well fitted empirical potential. We investigated the caloric curves of icosahedron nanoclusters and the significant depression in the melting temperatures of the copper nanoclusters was compared with that of the bulk copper. A structural transitions from decahedral to icosahedral shapes were shown. As the cluster size increased, the melting temperature increased, and the latent heat increased but seem to be saturated. However, the specific heat was unrelated to the cluster size.
\end{abstract}

Keywords : Icosahedron cluster, Melting properties, Molecular dynamics simulation

\section{INTRODUCTION}

Phase transitions in bulk materials have been studied since a very long time. In recent, the investigations on the thermal stability of atoms and molecular clusters have been performed, as it is vital to the possible application of the size-confined materials. Numerous work have been done both in experiments and in theoretical studies to clarify the thermodynamics behavior of clusters with different bonding types (van der Waals, covalent, ionic and metallic) [1-6]. Extended studies show that the reduction of melting temperature for some metal cluster is much smaller than that of Lennard-Jones cluster, and may be different from each other in separate metals such as $\mathrm{Co}$ and $\mathrm{Au}[4-6]$. Their nanoscopic properties differ in some main aspects from the bulk counterparts. One of the most important findings about the melting of clusters is the decrease of melting points with reduction nanocluster size, which is mainly due to the large percentage of atoms on the surface. Since atoms in clusters have fewer nearest neighbours and are thus weaker bound and less constrained in their thermal motion $[2,3]$, the latent heat of fusion is reduced, which is also due to a surface effect.

The shapes of metal nanoclusters have many types as follows: Trigonal bypyramid, pentagonal bypyramid, octahedron, truncated octahedron, capped decahedron, dodecahedron, tricapped trigonal prism, Icosahedron, etc. [7]. Among these, the most spherical shape of nanoclusters for metals with $f c c$ structure is icosahedral shape that has the multiple twinned boundaries and is composed of twenty deformed tetrahedra. All facets of the tetrahedra in the icosahedral clusters are $\{111\}$ surfaces, and these icosahedral nanolcusters have been found in previous atomic-scale simulations [7-16] and experiments [17-20]. Recently, Li et al. [14] investigated the structural transition from the cuboctahedral to icosahedral isomers for $\mathrm{Au}_{55}$ cluster due to the thermal vibrations of atoms. Garzón et al. [16] showed that the icosahedron $\mathrm{Au}_{55}$ is more stable than cuboctahedron $\mathrm{Au}_{55}$. However, little has been done to reveal the possible difference of the melting properties among an icosahedral nanocluster series. Therefore, we choose five icosahedral copper nanoclusters as shown in Table 1 . In this paper, we present results of molecular dynamics (MD) simulations on the icosahedral copper clusters. Beside the melting temperature, we also studied the latent heat of fusion which is sensitive to the size of system as well as the interaction properties of the materials.

\section{METHODS}

A semi-empirical potential with its attractive term based on a second-moment approximation of tightbinding (SMA-TB) model has been developed to describe the interaction of transition metals. The SMA is 
Table 1. Number of atoms, diameter, melting temperature and latent heat of simulated icosahedron nanoclusters.

\begin{tabular}{cccc}
\hline $\begin{array}{c}\text { Number of } \\
\text { atoms }\end{array}$ & $\begin{array}{c}\text { Diameter } \\
(\AA)\end{array}$ & $\begin{array}{c}\text { Melting } \\
\text { temperature }(\mathrm{K})\end{array}$ & $\begin{array}{c}\text { Latent heat } \\
(\mathrm{eV} / \mathrm{atom})\end{array}$ \\
\hline 13 & 5.74 & - & - \\
55 & 9.55 & 381 & 0.041 \\
147 & 14.32 & 556 & 0.064 \\
309 & 19.12 & 592 & 0.071 \\
561 & 23.96 & 658 & 0.073 \\
\hline \hline
\end{tabular}

Table 2. Parameters of the SMA-TB potential for copper used in this work (from Ref. [19]).

\begin{tabular}{cccc}
\hline \hline$A(\mathrm{eV})$ & $\xi(\mathrm{eV})$ & $p$ & $q$ \\
\hline 0.0855 & 1.224 & 10.960 & 1.189 \\
\hline \hline
\end{tabular}

not suitable, in principle, for noble metals where the $d$ band is full. However, it has been shown that the SMATB can give quite good agreement with experimental properties for noble metals as well as transition metals when the radial interaction range is sufficiently extended $[21,22]$. These results, together with the similarities between noble and transition metals in static and vibrational properties, confirm the feasibility of this model in noble metal systems $[4-6,15,16,23]$. The total potential energy of the system, $E_{t o t}$, can be expressed as a sum of the potential energies of each atom $i$. The energy of each atom, $E_{i}$, is expressed by the sum of the attractive band energy determined by the occupied local density of states, $E_{i}^{\text {hand }}$, and the Born-Mayer repulsive term determined by the ion core-core interaction, $E_{i}^{\text {rep }}$. The total energy of system, $E_{t o t}$, can be written as:

$$
\begin{aligned}
E_{\text {rot }} & =\sum_{i} E_{i}=\sum_{i}\left(E_{i}^{r e p}+E_{i}^{\text {hatud }}\right) \\
E_{i}^{r e p} & =\sum_{j} A \exp \left[-p\left(r_{i j} / r_{0}-1\right)\right] \\
E_{i}^{\text {hand }} & =\left[\sum_{j} \xi_{0}^{2} \exp \left[-2 q\left(r_{i j} / r_{0}-1\right)\right]\right]^{1 / 2}
\end{aligned}
$$

where $r_{0}$ and $r_{i j}$ are the nearest-neighbor bond length in a perfect crystal and the distance between $i$ and $j$ atoms, respectively. $A, \xi, p$ and $q$ are adjustable parameters which are determined by fitting the calculated values of cohesive energy, lattice constant and elastic constants to the experimental values. The parameters are empirically obtained by fitting the elastic and structural properties of
Table 3. Calculated physical properties of $\mathrm{Cu}$ using the SMA-TB potential, in comparison with experimental data: The cohesive energy per atom $\left(E_{c}\right)$, the lattice constant $(a)$, the elastic constants $\left(C_{11}, C_{12}\right.$, and $\left.C_{44}\right)$, the bulk modulus $(<B>)$ and the melting temperature $\left(T_{m}^{b}\right)$.

\begin{tabular}{lcc}
\hline \hline & SMA-TB [19] & Experiment \\
\hline$E_{c}(\mathrm{eV})$ & -3.544 & $-3.544[34]$ \\
$a(\AA)$ & 3.615 & $3.615[34]$ \\
$C_{11}\left(10^{12} \mathrm{dyn} / \mathrm{cm}^{2}\right)$ & 1.76 & $1.76[35]$ \\
$C_{12}\left(10^{12} \mathrm{dyn} / \mathrm{cm}^{2}\right)$ & 1.12 & $1.12[35]$ \\
$C_{44}\left(10^{12} \mathrm{dyn} / \mathrm{cm}^{2}\right)$ & 0.82 & $0.82[35]$ \\
$<B>\left(10^{12} \mathrm{dyn} / \mathrm{cm}^{2}\right)$ & 1.42 & $1.42[35]$ \\
$T_{m}^{h}(\mathrm{~K})$ & 1490 & $1356[36]$ \\
\hline
\end{tabular}

bulk material, as shown in Table 2. The cut off distance is set to the value between the fourth and fifth nearest neighbours of perfect crystal. Table 3 shows the calculated various physical properties of $\mathrm{Cu}$ using the SMA-TB potential, in comparison with experimental data.

A code base on constant temperature molecular dynamics scheme has carried out all simulations in this paper and in previous works [24-29]. The MD code uses the velocity Verlet algorithm, Gunsteren-Berendsen thermostat to keep constant temperature, and neighbor lists to improve computing performance [30]. Icosahedral copper clusters were heated up in step via scaling the atomic velocities with zero total linear and angular momenta. On heating, temperature was increased from $300 \mathrm{~K}$ by $1 \mathrm{~K}$ interval. At each temperature, MD runs of $2 \cdot 10^{5}$ steps were made with a time step of 0.5 fs (total $100 \mathrm{ps}$ ).

Several statistics were observed to corroborate the solid-liquid transitions. The mean kinetic temperature $k_{B} T=[2 /(3 N-6)]\left\langle\sum_{i=1}^{N}\left(m v_{i}^{2} / 2\right)\right\rangle$, where the angular brackets denote averaging over time and $k_{B}$ is the Boltzman constant, supply the energy to atoms in clusters, and the caloric curves of the clusters with the kinetic temperature, as shown in Fig. 2, were obtained from the last $10^{3}$ steps. The specific heat $c$ related to the energy fluctuation of the system, as a function of the kinetic temperature, is defined in a conventional form as

$$
c=\frac{Q}{m_{c}\left(T_{f}-T_{i}\right)}=\frac{E_{f}-E_{i}}{m_{c}\left(T_{f}-T_{i}\right)},
$$

where $Q$ is transferred internal energy, $m_{c}$ is the mass of cluster, $E_{f}$ and $E_{i}$ are the final and initial internal energies, and $T_{f}$ and $T_{i}$ are the final and initial kinetic temperatures, respectively. 


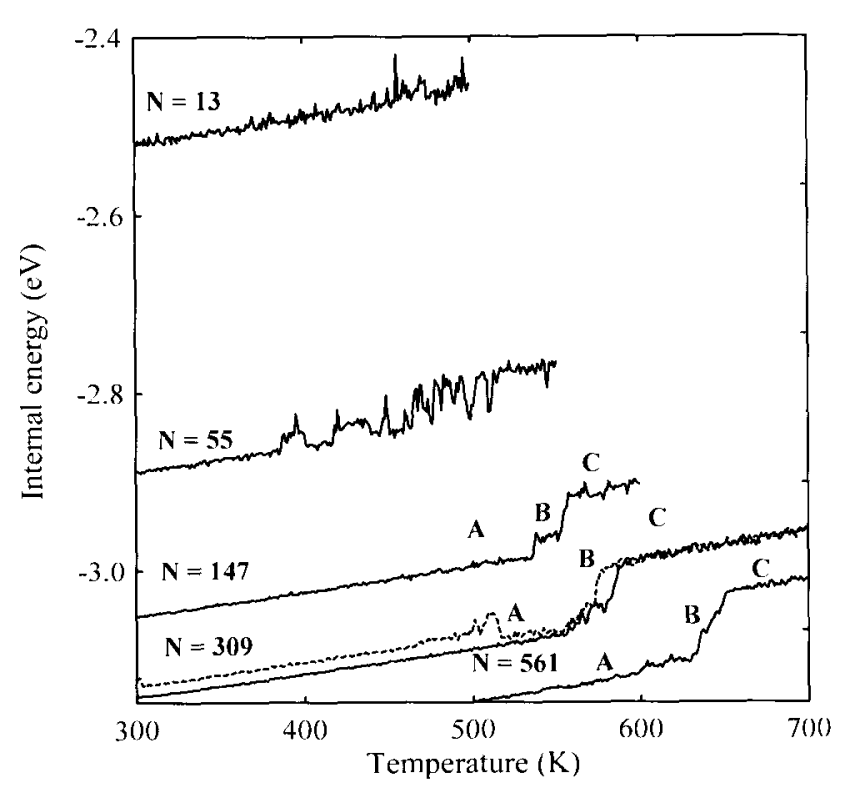

Fig. 1. Caloric curve as a function of temperature for the icosahedron clusters with $N=13,55,147,309$ and 561 atoms. Dashed line is the caloric curve for the decahedron cluster with $N=309$ atoms.

\section{RESULTS AND DISCUSSION}

Figure 1 shows the caloric curves of the clusters with temperature. We can also see from Fig. 1 the effect of size confinement on the melting properties of the clusters. The overall melting is clearly identified by the abrupt jump in the caloric curves except for the case of $\mathrm{Cu}_{13}$. The melting temperatures of the clusters are obviously lower than that of bulk material and Table 1 summarized the melting temperatures of the clusters obtained from the caloric curves. In the caloric curves for the clusters with $N=147,309$ and 561 atoms, labels A, B and C indicate the solid, transient and liquid states, respectively. In stage $\mathrm{A}$, the caloric curves increase linearly with increasing temperature, their slops correspond to the Dulong-Petit specific heat of the solid clusters, and the clusters remain their initial spherical shape as shown in Figs. 2(a) and 2(d). In stage B, the caloric curves increases exhibiting the upward curvatures prior to the melting transition. These are related to the loss of solid rigidity at the surface. The surface premelting of the clusters is apparently observed and shows the generation of defects, the vacancies or the interstitals on the surfaces of the clusters, as shown in Figs. 2(b) and 2(e). As the diameter of the clusters increases, thus transient phenomenon of the clusters is visibly observed. In the case of the clusters with $N=13$ and 55 atoms, since the size of the clusters is relatively small, the defects on the

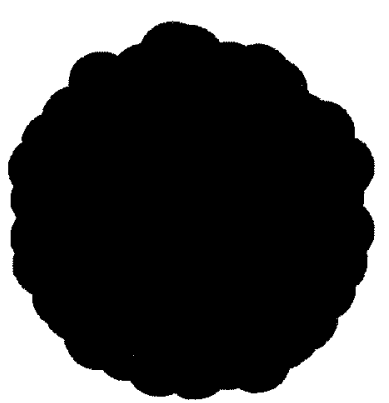

(a)

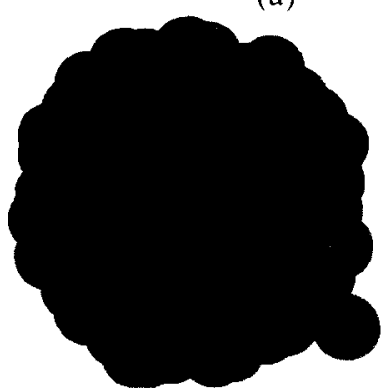

(b)

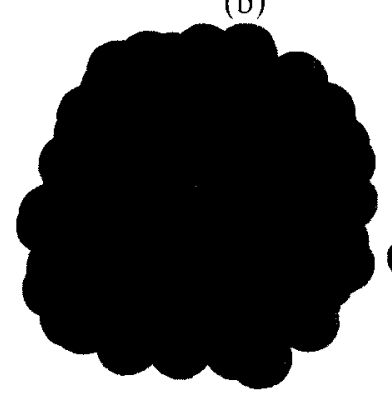

(c)

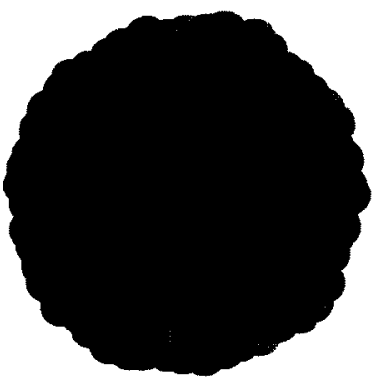

(d)

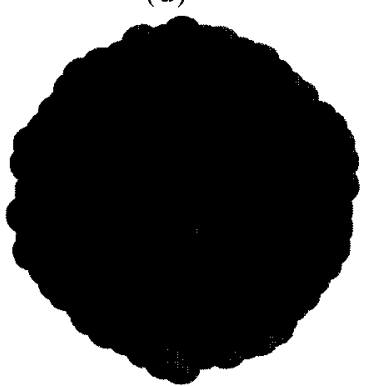

(e)

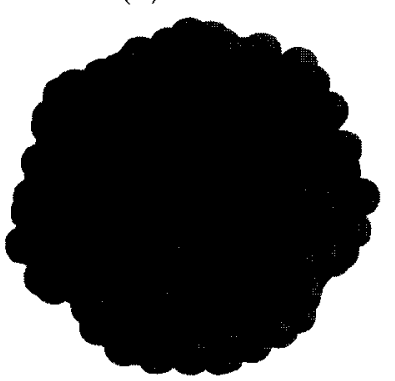

(f)
Fig. 2. Shapes of the clusters with $N=147$ atoms at (a) 500 , (b) 545 and (c) $560 \mathrm{~K}$ and with $N=309$ atoms at (d) 520 , (e) 575 and (f) $620 \mathrm{~K}$, respectively.

surfaces of the clusters are easier generated and recombined. Therefore, many variances of curvatures in the caloric curves are found in Fig. 1. Stage $C$ is after upward curvature in the caloric curves due to the latent heat during the melting transition. In this stage, the clusters are in the liquid state, as shown in Figs. 2(c) and $2(\mathrm{f})$, and the caloric curves increase again almost linearly with increasing temperature.

Figure 3 shows the radial distribution function $(r d f)$ and the angular correlation function $(a c f)$ with the size of cluster and the temperature. The $r d f$ and $a c f$ in stage A are similar to those of the solid state of the bulk material, and those in stage $\mathrm{C}$ are similar to those of the liquid of the bulk materials. Those in stage $B$, the transient state, are similar to those in stage $\mathrm{A}$, the solid state, because the clusters in stage $B$ have several defects on their surfaces. Li et al. [14] showed the structural transition from the cuboctahedral to icosahedral isomers for $\mathrm{Au}_{55}$ cluster due to the thermal vibrations of atoms and also 


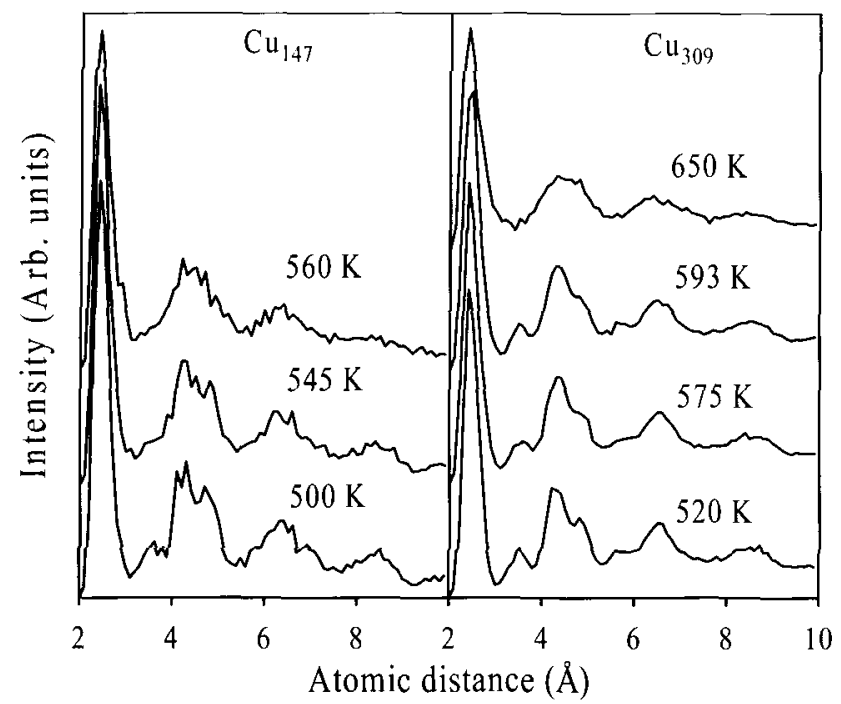

(a)

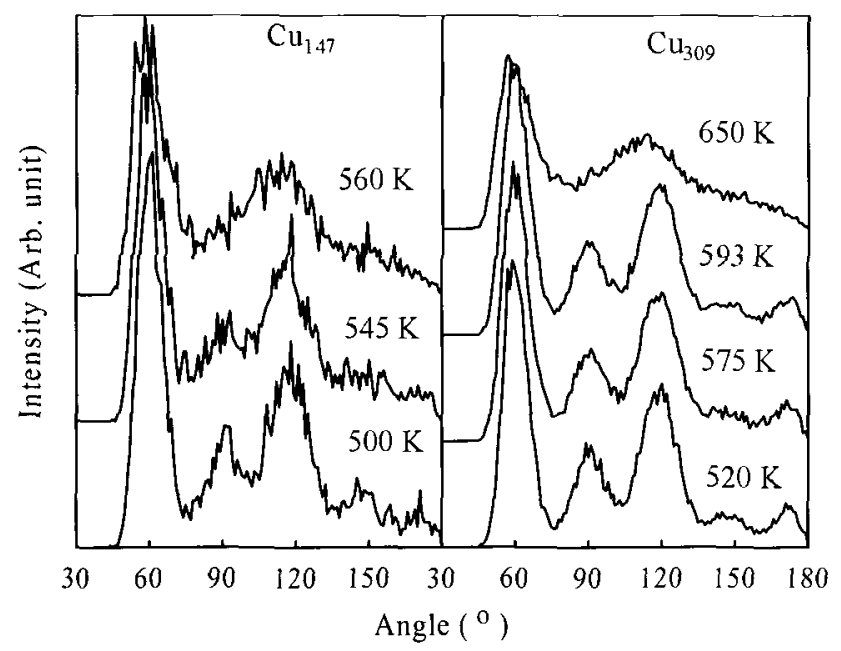

(b)

Fig. 3. (a) Radial distribution and (b) angular correlation functions with temperatures for the clusters with $N=$ 147 and 309 atoms.

showed another structural transition from a metastable icosahedral to another susceptible states with only a few atoms being rearranged. However, in our simulations of the icosahedral copper clusters with $N=55,147,309$ and 561 atoms, before their melting points, several defects on their surfaces were found but the structural transitions were not found. We also simulated the decahedron copper clusters with $N=13,55,147,309$ and 561 atoms. As the temperature of systems increased, the decahedron clusters were transformed into the icosahedron clusters, and as the diameter of the clusters increased, the temperature achieving the transformation of the decahedron into icosahedron increased. The dashed line in Fig. 1 indicates the caloric curve for the

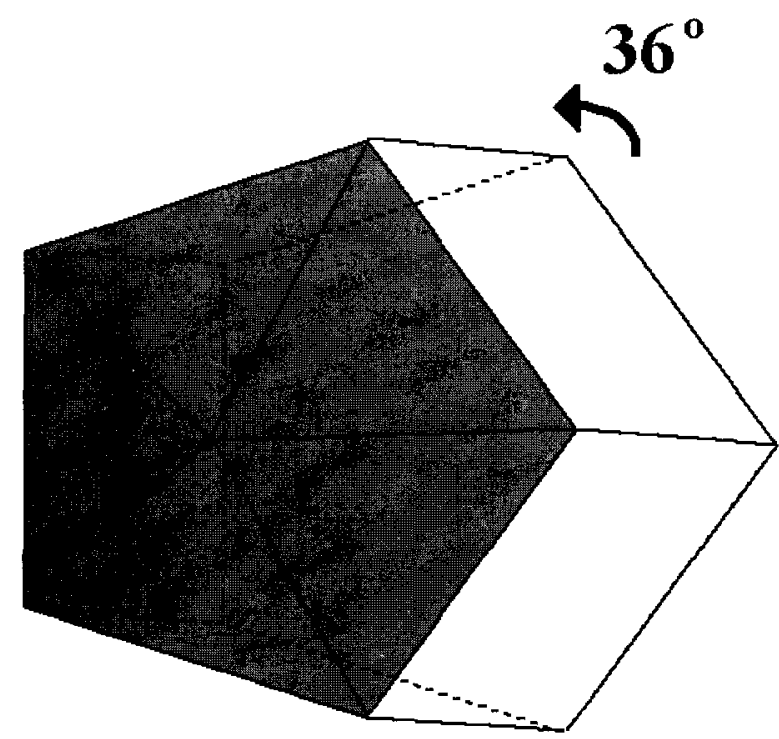

(a)

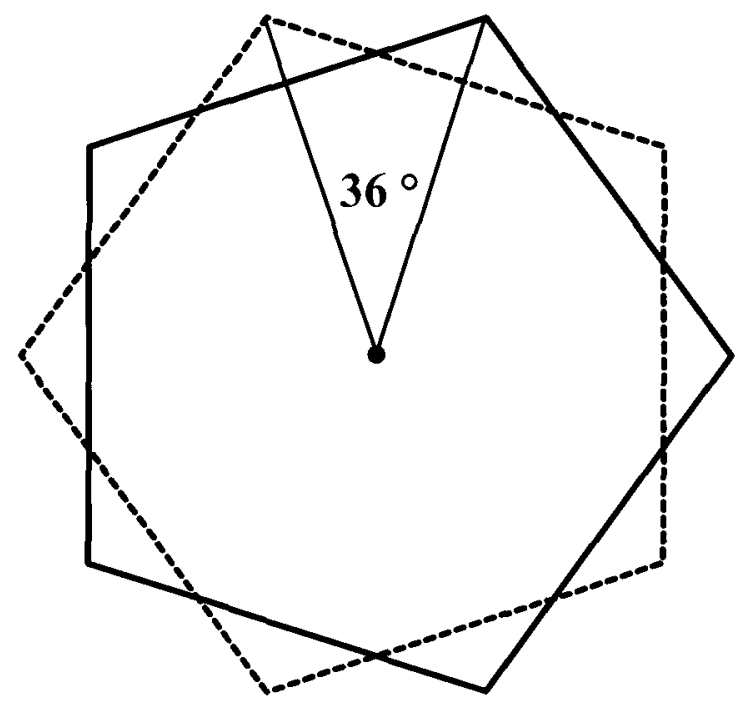

(b)

Fig. 4. (a) Decahedron. When a decahedron is twisted about $36^{\circ}$ due to shear stress, an icosahedron is formed as (b).

cluster with the initial decahedron cluster with $N=309$. The caloric curve shows that the decahedron cluster was transformed into an icosahedron cluster at $510 \mathrm{~K}$ and the melting temperature of this cluster case is lower $10 \mathrm{~K}$ than that of the icosahedron cluster with $N=309$ atoms. Figure 4(a) shows a decahedron composed of ten tetrahedra and five quadrangular pyramids. When a decahedron is twisted about $36^{\circ}$ due to shear stress, an icosahedron can be form as shown Fig. 4(b). The caloric curve of the decahedron cluster with $N=309$ atoms shows the small upward and downward curvatures when the structural transition is achieved. This shows when the decahedron clusters have the sufficient thermal energies 


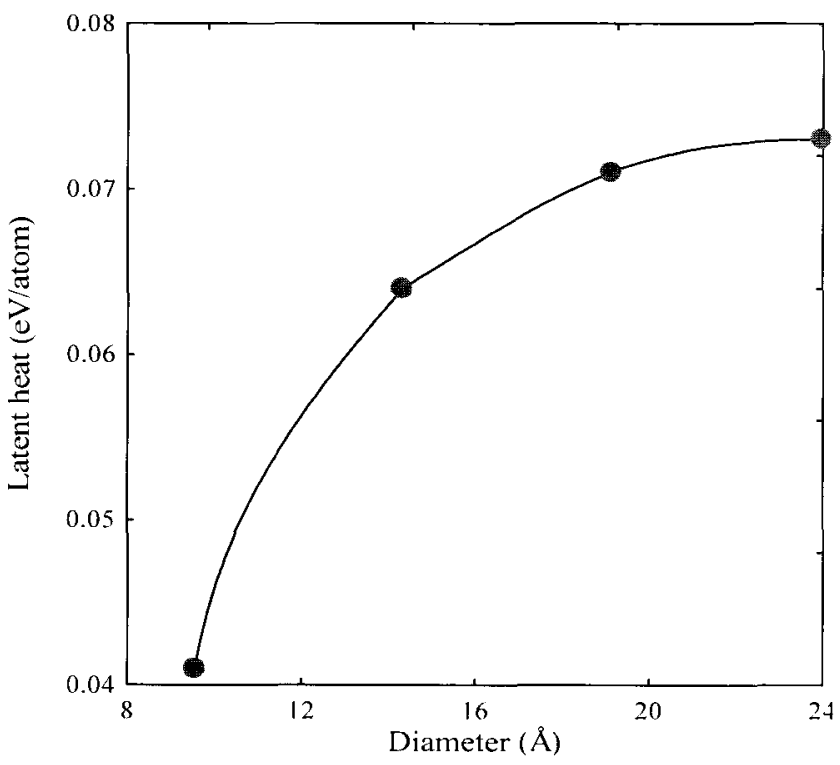

Fig. 5. Latent heats of the clusters as a function of the diameter of the cluster.

to be transformed into more stable icosahedron clusters, the structural transformations are achieved. As the diameter of the decahedron clusters increases, since the shear stress for the transformation increases, the temperature achieving the transformation increases. In the case of the decahedron cluster with $N=561$, the transformation of the decahedron into icosahedron was not achieved and the cluster reached the melting point with a decahedral shape. Ding et al. [31] showed that the central part of the pentagonal rod with multiple twin boundaries is highly compressed and sheared whereas the outer part is tensioned and the pentagonal rod structure is metastable. In previous MD simulations at $300 \mathrm{~K}$, the pentagonal needle-like nanorod with diameter $=15 \AA$ was transformed into a nanorod with an icosahedral model [32]. Therefore, we can see that the thermal energy of the fusion in the case of the decahedron cluster with $N=561$ is lower than the shear stress barrier required transforming the decahedron into icosahedron cluster.

We also calculated the specific heats of the clusters and the average specific heat of the clusters in this work is $0.3899 \mathrm{~J} / \mathrm{g}{ }^{\circ} \mathrm{C}$ during the solid state of the clusters, and this value is in good agreement with the experimental value at $25^{\circ} \mathrm{C}, 0.385 \mathrm{~J} / \mathrm{g}{ }^{\circ} \mathrm{C}$ [33]. During the melting transition, the specific heat curve shows a distinguished upward curvature and the specific heat in the liquid state is slightly higher than that in the solid state. This result shows that the specific heat of the icosahedral copper nanocluster is constant irrespective of the nanocluster size.

We also calculated the latent heat of the clusters, which is the energy to destroy the lattice at the melting temperature. Here it is measured as indicated in Fig. 1 as the height of the increases of the caloric curve near the melting temperature and is shown in Table 1 and Fig. 5. As the diameter of cluster increases, the latent heat of cluster increases and seems to be saturated in the cases of five icosahedron copper clusters. This work shows that the latent heat of the clusters is lower than that of the bulk copper, $0.1366 \mathrm{eV} /$ atom [33], due to a surface effect.

\section{SUMMARY}

In summary, we studied the size confinement effect on the properties of melting-like transition of small icosahedral copper clusters. The atomic interaction was described by the SMA-TB potential and classical molecular dynamics simulations were performed. We investigated the caloric curves of icosahedron nanoclusters and the significant depression in the melting temperatures of the copper nanoclusters was found compared with that of the bulk copper. A structural transition, that is, the transformation from decahedral to icosahedral shapes, was shown. As the cluster size increased, the melting temperature increased, and the latent heat increased but seem to be saturated. However, the specific heat was unrelated to the cluster size.

\section{ACKNOWLEDGMENTS}

This Research was supported by the Chung-Ang University Research Grants in 2002.

\section{REFERENCES}

[1] R. Kusche, Th. Hippler, M. Schmidt, B. von Issendorff, and Haberland H., "Melting of free sodium clusters", Eur. Phys. J. D, Vol. 9, p. 1, 1999.

[2] S. Carnalla, A. Posada, and I. L. Garzón, "Vibrational properties of nickel and gold clusters", Nanostructured Materials, Vol. 3, p. 385, 1993.

[3] M. Schmidt, R. Kusche, B. Issendorff, and H. Haberland, "Irregular variations in the melting point of size-selected atomic clusters", Nature, Vol. 393, p. $238,1998$.

[4] J. Jellinek, T. L. Beck, and R. S. Berry, "Solidliquid phase changes in simulated isoenergetic $\mathrm{Ar}_{13}$ ", J. Chem. Phys., Vol. 84, p. 2783, 1986.

[5] J. Jellinek and I. L. Garzón, "Melting of gold clusters", Z. Phys. D, Vol. 20, p. 239, 1991.

[6] I. L. Garzón and J. Jellinek, "Structural and dynamical properties of transition metal clusters", $Z$. 
Phys. D, Vol. 20, p. 235, 1991.

[7] N. T. Wilson and R. T. Johnston, "Modelling gold clusters with an empirical many-body potential", Eur. Phys. J. D, Vol. 12, p. 161, 2000.

[8] K. Michaelian, N. Rendon, and I. L. Garzon, "Structure and energetics of $\mathrm{Ni}, \mathrm{Ag}$, and $\mathrm{Au}$ nanoclusters", Phys. Rev. B, Vol. 60, p. 2000, 1999.

[9] S. Erkoc and T. Yilmaz, "Molecular-dynamics simulations of silver clusters", Physica E, Vol. 5, p. $1,1999$.

[10] S. Erkoc, "Stability of gold clusters: moleculardynamics simulations", Physica E, Vol. 8, p. 210, 2000.

[11] T. X. Li, S. Y. Yin, Y. L. Ji, B. L. Wang, G. H. Wang, and J. J. Zhao, "A genetic algorithm study on the most stable disordered and ordered configurations of $\mathrm{Au}_{38-55}$ ", Phys. Lett. A, Vol. 267, p. 403, 2000.

[12] C. R. A. Catlow, V. L. Bulatov, and R.W. Grimes, "Computational studies of the structures, energetics and dynamics of clusters", Nucl. Instru. Meth. Phys. B, Vol. 122, p. 301, 1997.

[13] L. Rongwu, P. Zhengying, and H. Yukun, "Molecular-dynamics simulations of slow copper cluster deposition", Phys. Rev. B, Vol. 53, p. 4156 , 1996.

[14] T. X. Li, S. M. Lee, S. J. Han, and G. H. Wang, "Structural transitions of Au55 isomers", Phys. Lett. A, Vol. 300, p. 86, 2002.

[15] I.L. Garzón and A. Posada-Amarillas, "Structural and vibrational analysis of amorphous $\mathrm{Au}_{55}$ clusters", Phys. Rev. B, Vol. 54, p. 11796, 1996.

[16] I. L. Garzón, K. Michaelian, M. R. Beltrán, A. Posada-Amarillas, P. Ordejón, E. Artacho, D. Sánchez-Portal, and J. M. Soler, "Lowest energy structures of gold nanoclusters", Phys. Rev. Lett., Vol. 81, p. $1600,1998$.

[17] B. Pauwls, G. van Tendeloo, W. Nouwen, L. T. Kuhn, P. Lievens, H. Lei, and M. Hou, "Lowenergy-deposited Au clusters investigated by highresolution electron microscopy and molecular dynamics simulations", Phys. Rev. B, Vol. 62 p. 10383, 2000.

[18] G. Canizal, J. A. Ascencio, J. Gardea-Torresday, and M. J. Yacaman, "Multiple twinned gold nanorods grown by bio-reduction techniques", J. Nanoparticle Research, Vol. 3, p. 475, 2001.

[19] Z. R. Dai, S. Sun, and Z. L. Wang, "Shapes, multiple twins and surface structures of monodisperse FePt magnetic nanocrystals", Surf. Sci., Vol. 505, p. 325, 2002.

[20] M. José-Yacamán, M. Marín-Almazo, and J. A. Ascencio, "High resolution TEM studies on palladium nanoparticles", J. Mol. Catalysis A: Chemical, Vol. 173, p. 61, 2001.

[21] F. Cleri and V. Rosato, "Tight-binding potentials for transition metals and alloys", Phys. Rev. B, Vol. 48 , p. 22, 1993.

[22] G. D'Agostino, A. Pinto, and S. Mobilio, "Simulated gold clusters and relative extended $\mathrm{x}$ ray-absorption fine-structure spectra", Phys. Rev. B, Vol. 48, p. 14447, 1993.

[23] J. M. Soler, M. R. Beltrán, K. Michaelian, I. L. Garzón, P. Ordejón, D. Sánchez-Portal and E. Artacho, "Metallic bonding and cluster structure", Phys. Rev. B, Vol. 61, p. 5771, 2000.

[24] J. W. Kang and H. J. Hwang, "Molecular dynamics simulation study on the melting of ultra-thin copper nanowires", J. Korean Phys. Soc., Vol. 40, p. 946, 2002.

[25] J. W. Kang and H. J. Hwang, "Molecular-dynamics study of the interaction between energetic $\mathrm{Al}$ clusters and an Al surface", Phys. Rev. B, Vol. 64, p. $014108,2001$.

[26] J. W. Kang, K. S. Choi, K. R. Byun, and H. J. Hwang, "Molecular dynamics study of $\mathrm{Al}$ atom and Al55 cluster deposition on $\mathrm{Al}$ substrate", J. Korean Phys. Soc., Vol. 36, p. 248, 2000.

[27] J. W. Kang and H. J. Hwang, "Pentagonal multishell Cu nanowires", J. Phys. : Condens. Matter, Vol. 14, p. 2629, 2002.

[28] J. W. Kang, K. S. Choi, J. C. Kang and H. J. Hwang, "Molecular dynamics simulations of film growth by energetic aluminum cluster impact", J. Korean Phys. Soc., Vol. 38, p. 158, 2001.

[29] J. W. Kang and H. J. Hwang, "Molecular dynamics simulations of ionized cluster beam deposition: case of study of aluminum", Comp. Mater. Sci., Vol. 21, p. $509,2001$.

[30] M. P. Allen and D. J. Tildesley, "Computer Simulation of Liquids", Clarendon press, Oxford, 1987.

[31] F. Ding, H. Li, J. Wang, W. Shen, and G. Wang, "Elastic deformation and stability in pentagonal nanorods with multiple twin boundaries", J. Phys. : Condens. Matter, Vol. 14, p. 113, 2002.

[32] J. W. Kang and H. J. Hwang, "Atomic scale simulations of polyhedral $\mathrm{Cu}$ nanorods", Nanotechnology, Vol. 13, p. 524, 2002.

[33] D. Halliday, R. Resnick and J. Walker, "Fundamentals of Physics", Extended Fifth Edition, John Wiley \& Sons, Inc., 1997.

[34] C. Kittel, "Introduction to Solid State Physics", Wiley, New York, 1966.

[35] G. Simmons and H. Wang, "Single Crystal Elastic Constants and Calculated Aggregated Properties", MIT press, Cambridge, 1971.

[36] R. Hultgren, P. D. Desai, D. T. Hawkins, M. Gleiser, and K. K. Kelly, "Selected Values of the Thermodynamics Properties of Binary Alloys", American Society for Metals, Metal Park, 1973. 